\title{
Erratum
}

\section{An extended LINMAP method for MAGDM under linguistic hesitant fuzzy environment}

Yun-Zhi Liu*, Zhi-Ping Fan and Guang-Xin Gao

[Journal of Intelligent \& Fuzzy Systems, 30(5) (2016), 2689-2703, 2016]

When this article was originally published, the affiliation of the authors is given incorrectly. Where it reads on page 2689:

Department of Information Management and Decision Sciences, Northeastern University of School of Business Administration, Shenyang, China

it should have read:

Department of Information Management and Decision Sciences, School of Business Administration, Northeastern University, Shenyang, China

The same correction applies to the corresponding author footnote on page 2689 where it reads:

*Corresponding author. Yun-Zhi Liu, Department of Information Management and Decision Sciences, Northeastern University of School of Business Administration, Shenyang 110167, China.

${ }^{*}$ Corresponding author. Yun-Zhi Liu, Department of Information Management and Decision Sciences, School of Business Administration, Northeastern University, Shenyang 110167, China.

The original article can be viewed here: https://dx.doi.org/10.3233/IFS-152022 